Health \& Medicine | Karla Hemming \& Monica Taljaard

\section{When is a stepped-wedge cluster randomised trial a good design choice?}

andomised trials are often used $\quad$ increase in life expectancies. Medical to reduce bias when testing new
medical treatments. Participants are randomly allocated to either the treatment group, where they receive the new treatment or experimental drug, or the control group, where they receive the current standard of care or any differences in patient outcomes, such as how long they sunive after a cancer diagnosis, can be attributed to experimental drug and all other possible explanations can be ruled out. Without randomisation, for instance, if doctors were to decide who should be treated with the experimental drug, any apparent differences in outcomes could be attributed to other disparities among the patients, rather than whether or not they were treated with the experimental drug.

Over the past decades, the use of clinical trials has undoubtably led to an

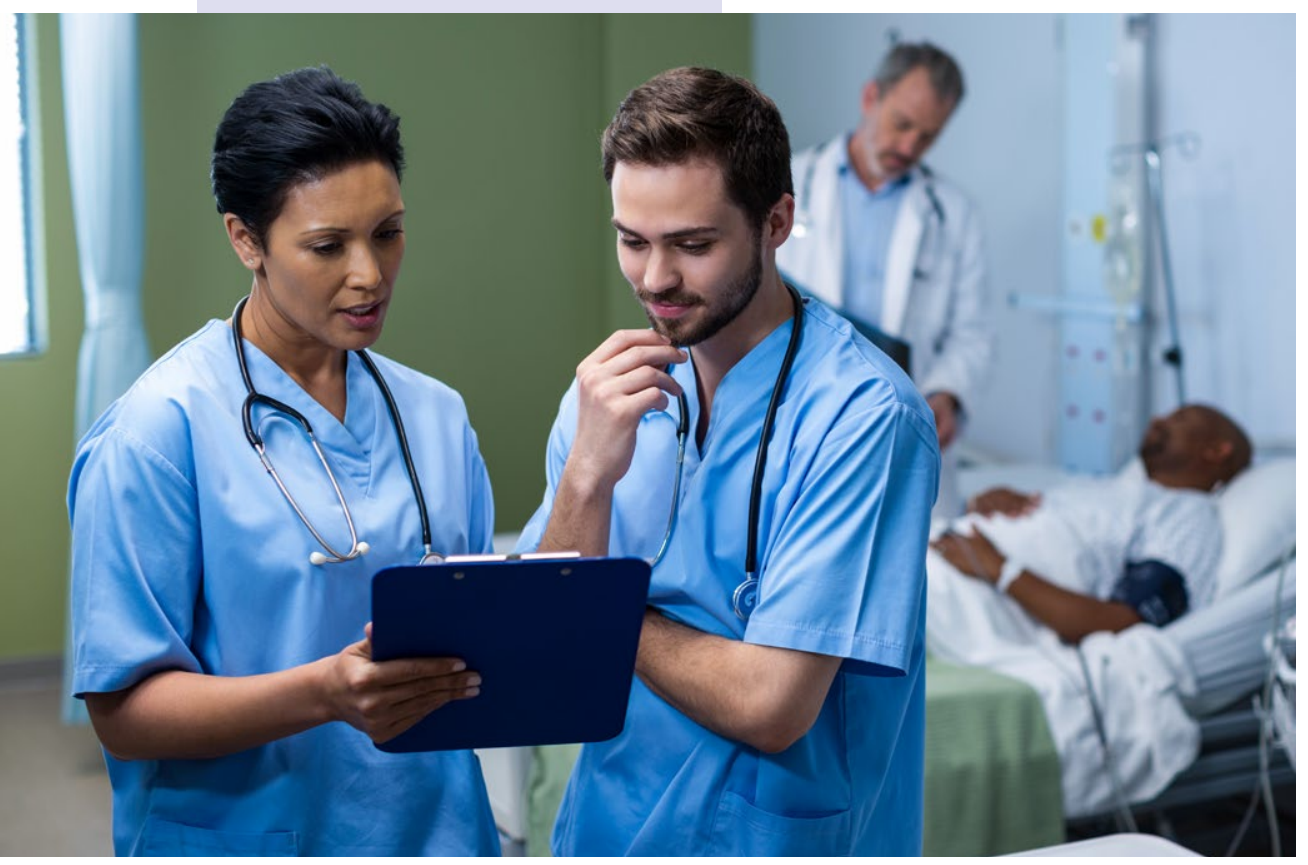
Precisely how this care is organised and delivered is also significant. For instance, it has been hypothesised tha doctors are permitted to work junior week could decrease the number of errors made. Nevertheless, policy for logistics and feasibility of conducting such studies is often perceived to be insurmountable. For example, while recruiting 1,000 participants for a patient randomised trial is not an easy task; translating this into recruiting 1,000 hospitals that would allow researchers to randomly decide whether junior doctors" otas are to be limited is infeasible.

et, Prof Karla Hemming (University of examine a new type of study design that brings hope and the chance to mprove the evidence base on which these policy decisions are made. This study design is the stepped-wedge cluster randomised trial (SW-CRT). Through an exploration of SW-CRT, they ascertain when this method is preferable to other study designs and

CLUSTER RANDOMISED TRIAL The cluster randomised trial is an established study design for pragmatic research, where the study can take place in real-world or typical practice settings. This is particularly usefur for the pragmatic evaluations of health policy interventions, including type interventions.

A cluster randomised controlled trial nvolves the randomisation of groups, clusters of subjects, such as hospita public health units or communities, ther than individual participants. In a paralle cluster randomised trial (paralleSigned to the intervention conditio while the other half are assigned to the control condition.

THE STEPPED-WEDGE CLUSTER RANDOMISED TRIAL In a SW-CRT, the clusters move sequentially from control to intervention conditions in a randomised order. This fis with what happens in a conventional Ingistical constrain will pre usully, some eceiving treatment simultaneously so the clusters tend to receive the treatment in steps or waves. A stepped wedge describes the shape that is produced Birmingham) and Prof Monica Taljaard why care should be taken when using it. 1). The crossover St intervention, In a SW-CRT, the new policy under evaluation is gradually and randomly rolled out to all hospitals, or clusters, Often, this desicn is universal. ethically advantageous, since it and educational or public health

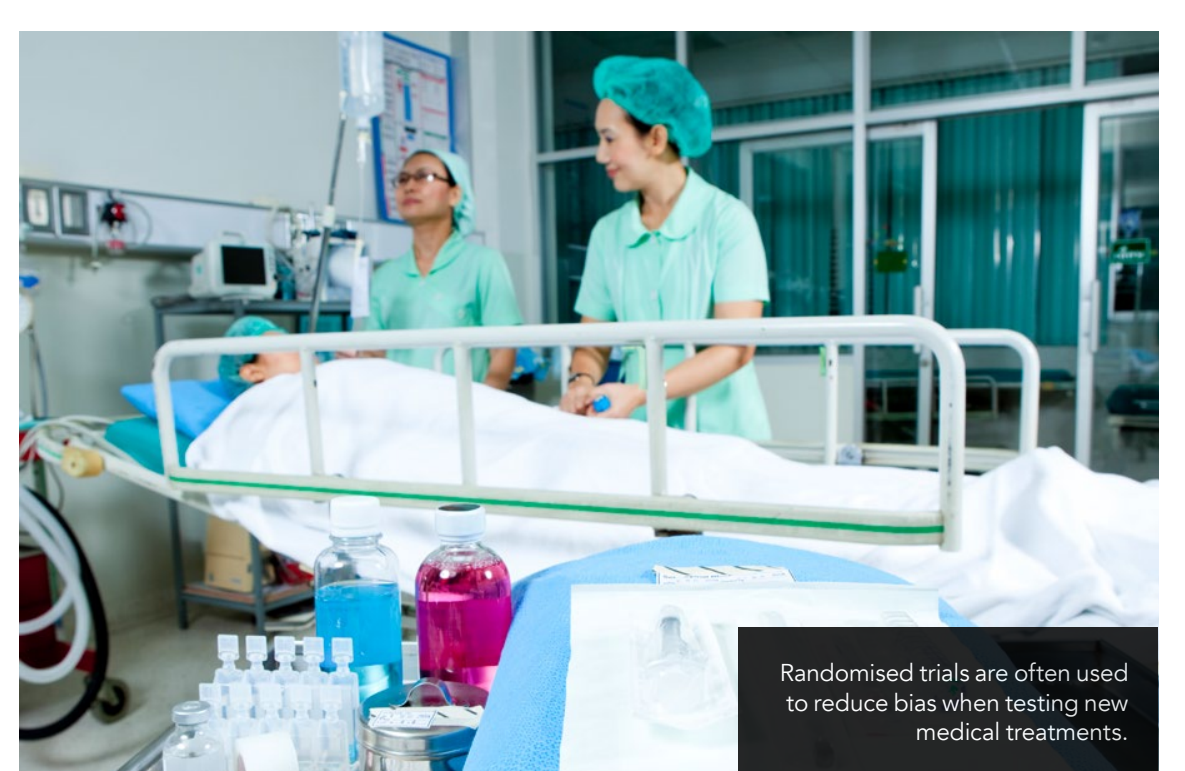

novel intervention eventally Novel interventions, however, don't always harm, which is why we want to evaluate them in the first place. There has been a surge in the use of the steppedwedge design for both service deliven

greater risks of bias because of the staggered nature of the roll-out, in that the observations under the control condition are collected earlier than those under the intervention condition. there is a natural tendency for practices to gradually improve or

illustration of the There has been a surge in the use of the becomes difficult to design (see figure stepped-wedge design for both service separate the effect of usually from control delivery and policy evaluations. the intervention. Any

and once it has been implemented, the and policy evaluations due to this intervention is not removed. perceived ethical advantage. However, this increase in uptake hasn't always been appropriate - and it is starting to be used when the alterative part $\begin{array}{lll}\text { ethically advantageous, since it } & \text { When compared with the conventional } & \text { parallel-CRT. These include bias that } \\ \text { allows all of the clusters to receive the } & \text { parallel-CRT, the SW-CRT is at } & \text { occurs when data collected under }\end{array}$

GREATER RISK OF BIAS

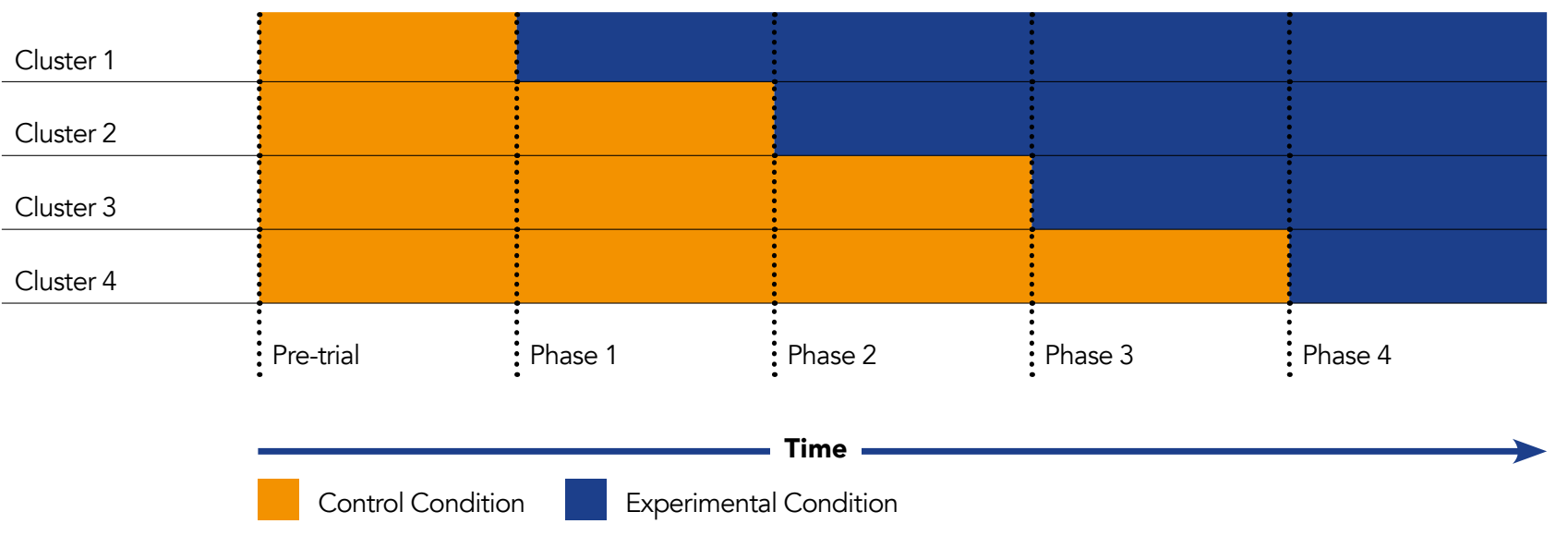

Figure 1. A pictorial representation of a stepped wedge trial: the clusters move
a randomised order. The intervention will gradually be rolled out in all clusters. minimise confounders,

is explain the observed results other than the treatments being studied. Conversely, the SW-CRT induces a Conversely, the SW-CRT induces a Confounder by design. Moreover, the 
the control condition becomes contaminated by the intervention condition, or vice versa. This is knon Within-cluster contamination is more likely to occur in a SW-CRT, since every cluster is exposed to both control and intervention conditions. Such bias underpins the development of the CONSORT (Consolidated Standards of Reporting Trials) extension for stepped-wedge cluster randomise trials. This reporting guideline highlights the additional complexities of the design and requires that investigators provide a clear justification for using this design. WHEN IS A STEPPED-WEDGE CLUSTER RANDOMISED TRIA

Methodologists are increasingly the use of SW-CRT Prof Heming and Prof Taljard have explored a number of situations and reveal potential conditions where a SW-CRT might be an appropriate choice. These justifications often overlap.

Often, interventions are rolled out without any robust randomised evaluation. Limited resources or capacity can mean that the roll-out is staggered. If the stakeholders, such as nurses, GPs or hospital management, can be persuaded to randomise the roll-out, a SW-CRT can be carried out and provide a means to conduct an be possible.

Permission to carry out cluster randomised trials is often required from gatekeepers, such as generat practice managers, ward matrons and lead consultants. They can be reluctant to participate in a trial unless they are assured that they will have the opportunity to receive the intervention which might be expected to offer some benefits and the expectation that the intervention is better than no intervention. Here, the SW-CRT enables cluster recruitment as it makes randomised evaluation more acceptable to cluster gatekeepers and

Due to pragmatic and logistical constraints, such as the roll-out of a scarce resource, the SW-CRT may be the only feasible design. While a parallel-CRT way, it becons in of the intervention is constrained to only a couple of clusters at a time.

The SW-CRT can have increased statistical power over other study designs, especially when the number of available clusters is restricted, due to availability, willingness to participate or limited trial budgets. Thus, an SWCRT may achieve the desired statistical power with fewer clusters than

Study designs may need to allow tim to realise the effect of the intervention. in the evalution of non straightow interventions, such as giving a drug to a patient, so the patient is thus exposed. When evaluating complex interventions, however, it might take considerable time for an intervention to become fully embedded and influence outcomes. While transition periods can be incorporated to allow for this delay, they might need to be quite long. This can increase the duration of the SW-CRT when compared with a parallel-CRT.

The stepped-wedge design is at risk of bias because of the staggered nature of the roll-out, particularly when there are only a small number of clusters.

OTHER CONSIDERATIONS Prof Hemming and Prof Taljaard explain There may be an imperative to provide that as the number of arguments in an evaluation of the intervention's favour of an SW-CRT increases, it is
effectiveness in a short amount of likely that the benefits of using the SW time, in which case the overall study CRT will outweigh its risks. They argue, duration can dictate the choice of however, that popularity and novelty trial method. Depending on the trial's should not be a factor in adopting the circumstances, the SW-CRT may take SW-CRT, and where a conventional more time than paralle-CRT, making parallel-CRT is feasible, it is likely to be the latter the preferred choice. the preferred design.

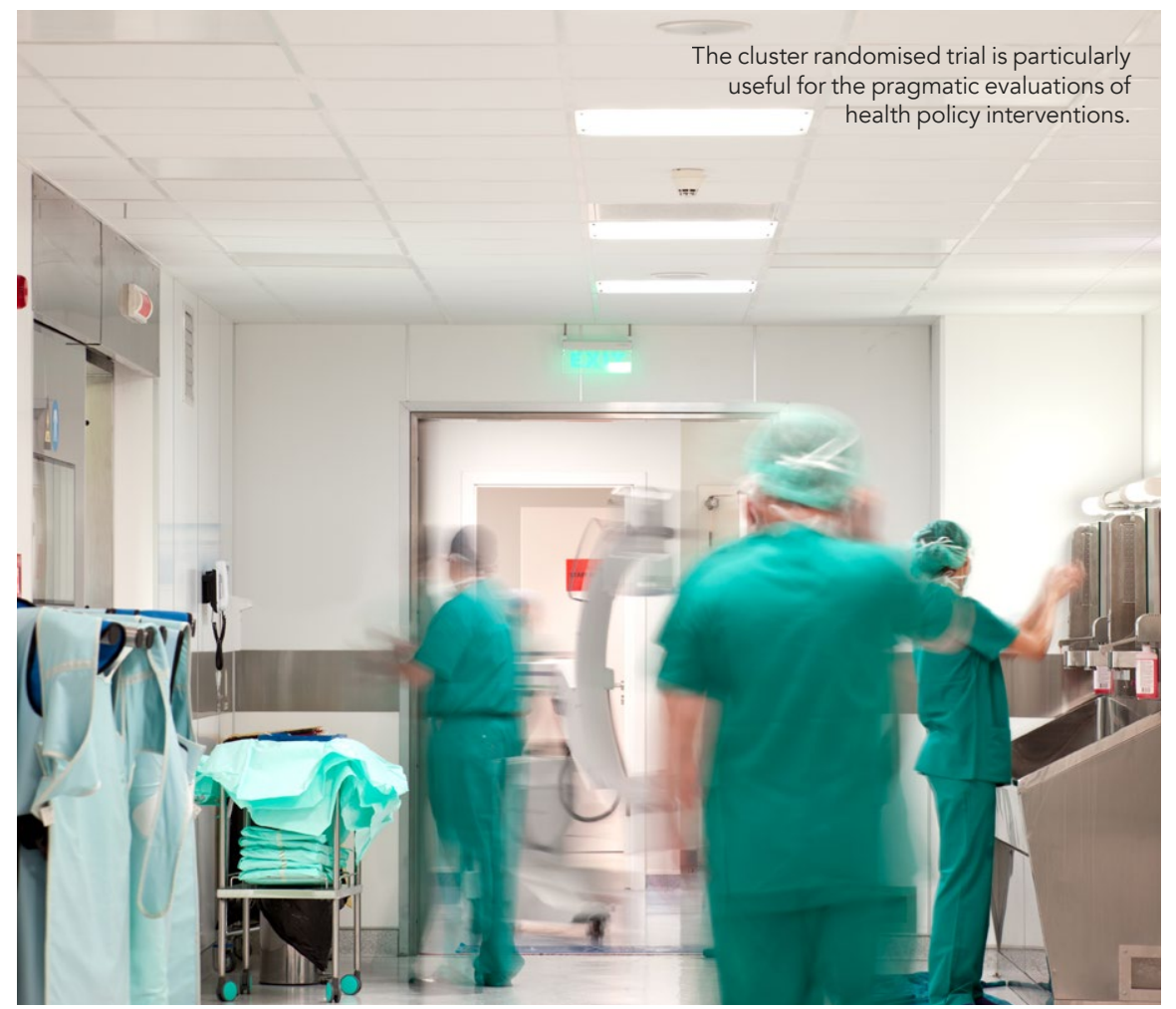

\section{Behind the Research}

\section{Prof Karla Hemming \& Prof Monica Taljaard}

E: k.hemming@bham.ac.uk T: +447387439884

Resources for stepped wedge and cluster trials: W: https://clusterrcts.shinyapps.io/rshinyapp/ W: https://clusterrandomisedtrials.qmul.ac.uk/

\section{Research Objectives}

Prof Hemming and Prof Taljaard examine the steppedwedge cluster randomised trial and determine stuations

\section{Detail}

\section{Address}

stitute of Applied Health Research

University of Birmingham, UK

Bio

Karla Hemming is Professor of Biostatistics, Institute of Applied Health Research, University of Birmingham. Prof Hemming's research interests include how to

design cluster and stepped-wedge trials in order to

aximise their statistical efficiency and minimise their isk of bias.

Monica Taljaard is Senior Scientist, Clinical Epidemiology Program Ottawa Hospital Research Institute. As a

Center, Dr Taljaard works with researchers and clinicians

from a variety of backgrounds in the design and analysis of cluster randomised trials, standard clinical trials, and observational studies.

Funding

This research was partly funded by the UK NIHR

Collaborations for Leadership in Applied Health Research

and Care West Midlands initiative.

Karla Hemming is funded by a NIHR Senior Research

Fellowship SRF-2017-10-002.

Collaborators

The Ottawa Hospital Research Institute Canada NHR $\mid \begin{aligned} & \text { National Institute } \\ & \text { for Health Research }\end{aligned}$

\section{References}

Hemming K., Taljaard M. (2020). Reflection on modern trial a good study design choice? International Journal of Epidemiology 49(3), 1043-1052 Available at. htps://doin org/10.1093/ije/dyaa077

Hemming K., Haines T.P., Chilton P.J., Girling A.J., Lilford R.J. (2015). The stepped wedge cluster randomised trial: rationale design, analysis, and reporting. BMJ, 350, h391.

Hemming K., Taljaard M., McKenzie J.E. et al. (2018). Reporting of stepped wedge cluster randomized trials. extension of the CONSORT 2010 statement with explanation and elaboration. BMJ, 363, k1614.

Huffman M.D., Mohanan P.P., Devarajan R. et al., Acute Coronary Syndrome Quality Improvement in Kerala (ACS Interntion on Clinica Outcomes in Patients inpovenent Acute Myocardia Infartion: The ACS QUIK Randomized Clinical Trial. JAMA, 319(6), 567-578.

\section{Personal Response}

What future extensions of the SW-CRT approach might further enhance its applicability?

II Policy decisions in health care need to be informed

by high-quality evidence. The stepped-wedge study, by

participating units, is appealing but is likely to face several.

important risks of bias. This is especially true when the

roll-out is across only a small number of units (e.g. hospitals. When the policy is implemented once-off at national level of participating units, observing what happens using an alternative design such as an interrupted time series is likely to be as, if not more, robust. 